VeritasE Scientia

Vol. 8. N 1.

Enero - Junio del 2019.

ISSN Edición Online: 2617-0639

https://doi.org/10.47796/ves.v8i1.106

\title{
RELACIÓN EXISTENTE ENTRE TIPOS DE LIDERAZGOS DIRECTIVOS Y LOS LOGROS DE LOS APRENDIZAJES EN ESCUELAS BÁSICAS MUNICIPALIZADAS PERTENECIENTES A CORPORACIÓN MUNICIPAL DE SOCIAL DE ANTOFAGASTA
}

\author{
EXISTING RELATIONSHIP BETWEEN TYPES OF LEADERSHIP LEADERSHIP AND ACHIEVEMENTS OF \\ LEARNING IN BASIC MUNICIPALIZED SCHOOLS BELONGING TO ANTOFAGASTA MUNICIPAL SOCIAL \\ CORPORATION
}

\author{
Ricardo Javier Díaz Asencio ${ }^{1}$ \\ Pedro Rosales Villarroel ${ }^{2}$ \\ Presentado: $18 / 04 / 2019$ \\ Aceptado: $17 / 06 / 2019$ \\ Publicado online:17/07/2019
}

\begin{abstract}
RESUMEN
Se estudió el estilo de liderazgo del Director(a) y si influenciaba o no el logro de los aprendizajes de sus estudiantes, encontrando en la evaluación SIMCE de los $4^{\circ}$ básicos, en la percepción que dan los puntajes entre los años 2012 a 2016 de las evaluaciones de Matemáticas y Comprensión Lectora, cuyos resultados son obtenidos de la página del SIMCE y son de conocimiento público; no así la forma de encontrar y determinar estilos de liderazgo direccional, los cuales fueron obtenidos en la medida de resultados de encuestas dirigidas a los diversos Directores(as) de escuelas pertenecientes a CMDS de Antofagasta, seleccionados al azar, utilizando el test de estilos de liderazgo de "Blake and Mouton", la cual es una traducción libre de Aroldo David Noriega, el que también se encuentra públicamente en una plataforma de internet, siendo este cruce de datos los indicadores para desarrollar la hipótesis sobre la influencia de este liderazgo versus los resultados académicos de los estudiantes, concluyendo que de alguna forma no se puede verificar dicha hipótesis en un $100 \%$, dado que los resultados tanto de una evaluación externa y los obtenidos vía encuesta no dan claridad a dicha afirmación, siendo en muchos casos positivos pero no constantes en el tiempo.
\end{abstract}

Palabras clave: liderazgo, dirección aprendizaje.

\footnotetext{
${ }^{1}$ Doctor en Educación con mención en Gestión Educativa

${ }^{2}$ Doctor, Director del Centro de estudios e investigaciones pedagógicas de la Facultad de Educación de la Universidad Autónoma de Chile
} 


\begin{abstract}
To the question of what to investigate, this study considered the question of Does the headmaster / headmistress' style have any influence or not in students' learning achievements. Using SIMCE evaluation in Fourth Graders as a reference to solve this question, overall the score interpretations of this test applied between 2012 and 2016 in Mathematics and Reading Comprehension sections. The results were taken from Simce webpage and they are part of public knowledge politics, however is not possible to find and determine leadership's styles, which were obtain from results of surveys directed to a different variety of headmasters and mistresses from schools that belong to Antofagasta CMDS, selected at random, using Blake and Mouton Leadership Style Test. This test is an Aroldo David Noriega's free translation, which can be found in an open internet platform. The crossing of this data becomes the indicators to develop the hypothesis that headmaster's / headmistresses' leadership style v/s student's academic results, concluding that this hypothesis cannot be verified in $100 \%$, due to the results of an external evaluation and results obtained through a survey do not show clarity of such hypothesis, in many cases, results were positive but not steady in time.
\end{abstract}

Keywords: leadership, learning direction.

\title{
INTRODUCCIÓN
}

Dentro de los establecimientos que dependen de la Corporación Municipal de Desarrollo Social de Antofagasta, que son cerca de 62, divididos en educación general básica y otros de educación media, existen diferentes tipos de formas de dirigir un establecimiento, unos con muy buenos resultados en lo académico, y otros con resultados en lo social, siendo los Directores(as) elegidos por Alta Dirección Pública los llamados a realzar los resultados de aprendizajes de sus escuelas. Por otro lado, la evaluación estandarizada llamada Simce, se da todos los años en diversos niveles de las escuelas, entregando datos duros para producir estudios o a lo menos interrogantes sobre cómo se está académicamente, siendo en este caso particular la evaluación de los $4^{\circ}$ años básicos la escogida para determinar la veracidad o no de la interrogante principal. Existe alguna bibliografía al respecto, como los estudios de comportamiento académico en establecimientos particulares, de corte subvencionado y otros no, como también estudios gubernamentales, evaluando efectos de liderazgo tanto emanados desde Dirección como desde las mismas aulas. También se han hecho análisis sobre cómo estos tipos de liderazgos van en desarrollo de nuevos planes y programas que son indicativos de un desarrollo de dichos aprendizajes.

Se define que todo estilo de liderazgo debe estar centrado en el aprendizaje de los alumnos(as), esto como principio básico de toda dirección organizativa de una escuela que busca que sus estudiantes aprendan, este hecho queda codificado y definido esencialmente por la OCDE, buscando la mejora de los conocimientos determinado por un liderazgo propio de cada líder pedagógico. Se hace necesario comprobar una directa relación en entre dichos aprendizajes y un estilo de liderazgo determinado, cuyos resultados académicos den fe en lo que se requiere o simplemente descartar dicha aseveración, lo cual puede ser indicativo de que no interesando el estilo los aprendizajes van de la mano otros elementos 
que potencian dichos logros académicos.

La Corporación Municipal de Desarrollo Social de Antofagasta (CMDS), tiene como misión mejorar la calidad de vida de las familias de Antofagasta, implementando servicios de educación y salud, a través de una gestión integrada, innovadora y eficiente. Administra trece liceos de Educación Media y treinta y siete escuelas de Educación General Básica, con aproximadamente cuarenta mil estudiantes. Cada escuela basa su estilo en directrices que se declaran en el Proyecto Educativo Institucional (PEI). En la actualidad, esta responsabilidad está debidamente asumida por estas autoridades, dado que lo exige en su postulación a directores a través de la Alta Dirección Pública (ADP). "El Simce está diseñado para evaluar la medida en que los estudiantes están aprendiendo", explicó Lorena Meckes (2016). El año anterior los resultados del Simce arrojó una baja de 15 puntos en la prueba de Lenguaje aplicada en octavos básicos. Así, los estudiantes obtuvieron un promedio de 240 puntos, en comparación con los 255 obtenidos en el test aplicado en 2013. En segundo medio también se registró una caída de dos puntos en Lectura, de 254 puntos en 2013 a 252 en la última medición. En segundos medios los alumnos obtuvieron 265 puntos, dos menos que en 2013. Para el curso medio en compresión lectora se indican 247 unidades, donde el resultado es menor al año pasado por cinco puntos. Tomando en cuenta los parámetros de 2010 y 2012, cayeron 12 puntos.

Ricardo Ponce Vidal (2008), refiere que "El estilo de Liderazgo Democrático desarrollado por los directores(as) impacta en los profesores de manera positiva", María Paola Sevilla Buitrón (2011), presentó un estudio emanado desde el Ministerio de Educación de Chile denominado: "Liderazgo directivo y resultados de los estudiantes" y muestra que es posible identificar equipos directivos más efectivos entre quienes postulan y ganan un incentivo. Humberto Hernán Riffo Muñoz (2014), concluye que "los equipos directivos de los establecimientos educacionales no jerarquizan las prácticas de gestión que son claves según el modelo de escuelas eficaces."

Gradualmente se ha ido abriendo paso en la sociedad chilena la convicción de que, para mejorar la efectividad de las escuelas y liceos y, por tanto, para elevar los logros de aprendizajes de nuestros alumnos, se requiere renovarla. Una reciente investigación realizada por UNICEF en Chile, las escuelas efectivas se caracterizan, ante todo, por el concepto de la buena gestión. Entre las claves de los buenos resultados que tienen raíz en la gestión pedagógica están el liderazgo directivo y técnico, proyectos educativos con metas concretas y priorizadas, reglas claras y explícitas, profesionalismo y ética del trabajo, planificación y evaluación, desarrollo profesional docente, compromiso e identidad institucional.

El liderazgo se define como la capacidad de convencer a los demás que la forma de gestionar es la adecuada para conseguir los objetivos propuestos como equipo. Los estilos no son únicos, sino, que dependiendo de la situación se desarrollan con más o menos precisión.

Un liderazgo para el aprendizaje toma como núcleo de su acción la calidad de enseñanza ofrecida y los resultados de aprendizaje alcanzados por los alumnos. El asunto prioritario es, qué prácticas de la dirección escolar crean un contexto para un mejor trabajo del profesorado y, conjuntamente, de todo el establecimiento educacional, impactando positivamente en la mejora de los aprendizajes del alumnado. Las prácticas de liderazgo han cambiado dramáticamente en las dos últimas décadas, particularmente en contextos de política educativa donde los centros educativos tienen mayor autonomía y, paralelamente, una mayor responsabilidad por los resultados escolares. La investigación internacional es convergente al señalar que el liderazgo directivo tiene el potencial de impactar sobre los resultados de aprendizaje de los estudiantes.

Los rasgos de las escuelas efectivas chilenas pueden servir de insumo para explicar por qué algunas escuelas mejoran hacia los estándares de excelencia, otras se mantienen, y algunas decaen. Identificar los rasgos que hacen que una escuela sea efectiva, fue una preocupación de los investigadores durante años. Estos esfuerzos derivaron finalmente en cinco claves, definidas como: presencia de buenos profesores, salas de clase adecuadas, buena relación familia - escuela, liderazgo del director y 
evaluación permanente.

El sistema nacional de evaluación de resultados de aprendizaje fue fundado en 1988 con el objetivo de institucionalizar diversas iniciativas en el ámbito de la evaluación que venían desarrollándose en Chile desde los años sesenta. Desde 2012, SIMCE pasó a ser el sistema de evaluación que la Agencia de Calidad de la Educación utiliza para evaluar los resultados de aprendizaje de los establecimientos. El Simce evalúa los logros de aprendizaje en las asignaturas de lenguaje y comunicación (Comprensión de Lectura y Escritura); matemática; ciencias naturales; historia, geografía, ciencias sociales e inglés. Las pruebas Simce se aplican a estudiantes de $2^{\circ}, 4^{\circ}, 6^{\circ}, 8^{\circ}$ básico, II y III medio, y se informa oportunamente a los establecimientos las asignaturas que serán evaluadas en el año en curso, en el nivel que corresponda. A partir de 2013, se aplican pruebas censales para estudiantes de $6^{\circ}$ básico con discapacidad sensorial. Esta evaluación se enmarca dentro de lo establecido en la legislación vigente en materia de igualdad de oportunidades e inclusión educativa de los estudiantes con discapacidad.

\section{OBJETIVOS}

a) Establecer tipos de liderazgo directivo en escuelas básicas de CMDS de Antofagasta.

b) Identificar logros de aprendizajes dentro de las escuelas básicas de CMDS de Antofagasta.

c) Relacionar tipos de liderazgo con logro de aprendizajes dentro de las escuelas básicas de CMDS de Antofagasta.

\section{METODOLOGÍA}

Es una investigación correlacional - explicativa, a través de la evaluación Simce y el estilo de liderazgo de los Directores(as) de los establecimientos involucrados en el estudio; y tiende a demostrar hechos de relaciones causa - efecto. Se tiene un grupo intervenido $(G)$ que hace referencia a los niveles de aprendizaje de los $4^{\circ}$ básicos en relación la variable del tipo de liderazgo (O) de cada Dirección que establece en los establecimientos educacionales correspondientes a CMDS. El ámbito hace referencia a instituciones educativas dependientes de la Municipalidad de Antofagasta, escuelas municipalizadas, en donde se imparte educación general básica. La población consta de 50 escuelas municipales de la ciudad de Antofagasta, todas pertenecientes a la Corporación Municipal de Desarrollo Social de la misma ciudad. La muestra fue estratificada de afijación proporcional y al azar, y estuvo representada por 17 escuelas que tienen educación general básica en sus establecimientos. La recolección de datos está sujeta al test de estilos de liderazgo de Blake and Mouton. La información para el cruce de datos se obtiene de los resultados anuales SIMCE de los $4^{\circ}$ básicos de escuelas pertenecientes a CMDS de Antofagasta, de los años 2012, 2013, 2014, 2015 y 2016, obtenidos desde la base de datos públicos de la página del Ministerio de Educación de Chile; de resultados de Lenguaje y Matemática respectivamente.

\section{RESULTADOS}

Dentro del análisis de los resultados cuantitativo, tabla 01, se determina que de 17 establecimientos seleccionados como muestra hay 10 pertenecientes al grupo socio económico medio y 7 en el medio bajo, obteniendo el estilo de liderazgo "Country Club" un Director(a) por grupo socio económico, no así el estilo "Líder de equipo" cuya representatividad es más uniforme, siendo el estilo predominante de dicha investigación, en un análisis más cualitativo, podríamos conjeturar que el estilo predominante se pudiese dar dado al elemento etario que poseen los nuevos Directores(as) de ADP en ejercicio. 
Tabla 1

Cuadro General resultados test de liderazgo

\begin{tabular}{|c|c|c|c|c|c|c|c|c|c|c|c|}
\hline \multirow{3}{*}{ 옹 } & & \multirow{3}{*}{ G.S.E. } & \multicolumn{3}{|c|}{ Contry Club } & \multicolumn{2}{|l|}{ Indeferente } & \multicolumn{3}{|c|}{ Lider de Equipo } & \multirow{3}{*}{$\begin{array}{l}\text { Autoritario } \\
\text { Personas Tareas } \\
\text { Alto Bajo Alto Bajo }\end{array}$} \\
\hline & & & \multicolumn{2}{|c|}{ Personas } & Tareas & Personas & Tareas & \multicolumn{2}{|c|}{ Personas } & \multirow{2}{*}{$\begin{array}{l}\text { Tareas } \\
\text { Alto Bajo }\end{array}$} & \\
\hline & & & $\overline{\text { Alto }}$ & Bajo & Alto & Bajo Alto Bajo & Alto Bajo & Alto & Bajo & & \\
\hline \multicolumn{12}{|c|}{ ESCUELA } \\
\hline 1 & Marta Narea & Medio & & 6,8 & 4,6 & & & & & & \\
\hline 2 & Japón & Medio & & & & & & & 7,8 & 6,6 & \\
\hline 3 & Gustav o Le Paige & Medio & & & & & & & 7,2 & 6,0 & \\
\hline & Italia & Medio & & & & & & & 7,2 & 6,8 & \\
\hline 5 & Jose Papic & Medio & & & & & & & 6,8 & 6,6 & \\
\hline 6 & Dario Salas & Medio & & & & & & & 7,6 & 6,8 & \\
\hline 7 & Alberto Hurtado & Medio & & & & & & 8,2 & & 8,0 & \\
\hline 8 & Arturo Prat & Medio & & & & & & & 7,6 & 7,4 & \\
\hline 9 & Armando Carrera & Medio & & 7,2 & 5,8 & & & & & & \\
\hline 10 & Las Rocas & Medio & & & & & & & 6,0 & 6,4 & \\
\hline 11 & Andres Sabella & Medio Alto & & & & & & 8,6 & & 8,6 & \\
\hline 12 & Edda Cuneo & Medio Alto & & & & & & & 7,8 & 7,8 & \\
\hline 13 & Libertadores de Chile & Medio Alto & & & & & & & 6,4 & 6,8 & \\
\hline 14 & España & Medio Bajo & & 6,0 & & 5,6 & & & & & \\
\hline 15 & Juan Pablo II & Medio Bajo & & & & & & 8,6 & & 8,2 & \\
\hline 16 & Patricio Cariola & Medio Bajo & & & & & & & 7,2 & 8,0 & \\
\hline 17 & Santiago Amengual & Medio Bajo & & & & & & 8,4 & & 6,2 & \\
\hline
\end{tabular}

Tabla 2

Resultados SIMCE Lenguaje $4^{\circ}$ básicos

\begin{tabular}{lcccccc}
\hline \multirow{2}{*}{ ESCUELAS } & 2012 & 2013 & 2014 & 2015 & 2016 & PROM \\
\cline { 2 - 7 } & 248 & 251 & 254 & 250 & 273 & 255 \\
\hline Marta Narea & 268 & 280 & 273 & 275 & 265 & 272 \\
Japón & 257 & 252 & 262 & 262 & 261 & 259 \\
Gustav o Le Paige & 236 & 258 & 242 & 233 & 247 & 243 \\
Italia & 241 & 242 & 238 & 228 & 246 & 239 \\
Jose Papic & 251 & 266 & 265 & 239 & 250 & 254 \\
Dario Salas & 271 & 243 & 256 & 253 & 270 & 259 \\
Alberto Hurtado & 248 & 252 & 249 & 263 & 272 & 257 \\
Arturo Prat & 259 & 232 & 245 & 250 & 247 & 247 \\
Armando Carrera & 253 & 227 & 235 & 242 & 270 & 245 \\
Las Rocas & 263 & 275 & 263 & 272 & 271 & 269 \\
Andres Sabella & 260 & 282 & 279 & 292 & 299 & 282 \\
Edda Cuneo & 260 & 241 & 262 & 248 & 254 & 253 \\
Libertadores de Chile & 238 & 218 & 231 & 224 & 241 & 230 \\
España & 238 & 229 & 240 & 248 & 240 & 239 \\
Juan Pablo II & 234 & 221 & 217 & 228 & 245 & 229 \\
Patricio Cariola & 262 & 248 & 244 & 261 & 245 & 252 \\
Santiago Amengual & & & & &
\end{tabular}


Tabla 03.

Resultados SIMCE Matemática $4^{\circ}$ básicos

\begin{tabular}{lccllll}
\hline \multirow{2}{*}{ ESCUELAS } & \multicolumn{7}{c}{ MATEMATICA } \\
\cline { 2 - 7 } & 2012 & 2013 & 2014 & 2015 & 2016 & PROM \\
\hline Marta Narea & 239 & 231 & 221 & 247 & 241 & 236 \\
Japón & 269 & 280 & 274 & 270 & 265 & 272 \\
Gustav o Le Paige & 250 & 253 & 254 & 258 & 265 & 256 \\
Italia & 243 & 229 & 224 & 209 & 238 & 229 \\
Jose Papic & 224 & 229 & 238 & 223 & 236 & 230 \\
Dario Salas & 240 & 270 & 258 & 204 & 224 & 239 \\
Alberto Hurtado & 250 & 228 & 230 & 234 & 256 & 240 \\
Arturo Prat & 248 & 261 & 249 & 269 & 291 & 264 \\
Armando Carrera & 259 & 227 & 219 & 235 & 232 & 234 \\
Las Rocas & 257 & 219 & 227 & 232 & 258 & 239 \\
Andres Sabella & 252 & 266 & 250 & 254 & 256 & 256 \\
Edda Cuneo & 261 & 296 & 272 & 294 & 303 & 285 \\
Libertadores de Chile & 243 & 211 & 224 & 221 & 253 & 230 \\
España & 244 & 220 & 226 & 226 & 235 & 230 \\
Juan Pablo II & 224 & 218 & 236 & 236 & 240 & 231 \\
Patricio Cariola & 241 & 223 & 214 & 225 & 238 & 228 \\
Santiago Amengual & 250 & 257 & 256 & 250 & 245 & 252 \\
\hline
\end{tabular}

La diferencia entre un puntaje y otro sobre el promedio de los resultados anuales entre las evaluaciones SIMCE de lenguaje y matemática se ven reflejada en la tabla 04.

Tabla 04.

Diferencia promedios anuales puntajes SIMCE

\begin{tabular}{|c|c|c|c|c|c|c|}
\hline & & & 2013 & 2014 & 2015 & 2016 \\
\hline 1 & Las Rocas & Lider de Equipo & -3 & -3 & 11 & 8 \\
\hline 2 & Andres Sabella & Lider de Equipo & 11 & -6 & -1 & -8 \\
\hline 3 & Gustavo Le Paige & Lider de Equipo & -1 & 5 & 2 & 3 \\
\hline 4 & Jose Papic & Lider de Equipo & 4 & -11 & -12 & 12 \\
\hline 5 & Dario Salas & Lider de Equipo & 3 & 2 & -12 & 15 \\
\hline 6 & Juan Pablo II & Lider de Equipo & 22 & -6 & -40 & 15 \\
\hline 7 & Edda Cuneo & Lider de Equipo & -25 & 7 & 1 & 19 \\
\hline 8 & Arturo Prat & Lider de Equipo & 9 & -8 & 17 & 16 \\
\hline 9 & Santiago Amengual & Lider de Equipo & -29 & 2 & 11 & -3 \\
\hline 10 & Libertadores de Chile & Lider de Equipo & -32 & 8 & 6 & 17 \\
\hline 11 & Japón & Lider de Equipo & 28 & -13 & 17 & 8 \\
\hline 12 & Italia & Lider de Equipo & -26 & 17 & -8 & 19 \\
\hline 13 & Patricio Cariola & Lider de Equipo & -7 & 14 & -4 & -2 \\
\hline 14 & Alberto Hurtado & Lider de Equipo & -3 & -3 & 6 & -11 \\
\hline 15 & Marta Narea & Country Club & 13 & -14 & 6 & 1 \\
\hline 16 & España & Country Club & -22 & 10 & -4 & 13 \\
\hline 17 & Armando Carrera & Country Club & -16 & -6 & 11 & 15 \\
\hline
\end{tabular}




\section{DISCUSIÓN}

No se observa el cumplimiento de una buena asociación entre liderazgo y resultados finales de aprendizaje, que estos resultados son fluctuantes anualmente, con algunos atisbos a subir o bajar, pero más sujetos al primer concepto, no siendo primordial el estilo que se ejecuta en el liderazgo de esos establecimientos. Puede ser que intervenga vagamente el estilo que da la Dirección en un tipo de conducta curricular, pero la variable interviniente de la metodología empleada por el docente de turno va a ser más profunda en una discusión que solamente el modelo de gestión que da el Director(a) de un establecimiento, siendo también vaga esta información debido a que puede ser un trabajo sistemático en el tiempo como azaroso en el concepto del perfil de los estudiantes que ese año contemplan rendir dicha evaluación SIMCE, que pudiesen ser en su mayoría trabajo del mismo colegio, como la incorporación, por diversos motivos, de estudiantes que van llegando de otros establecimientos educacionales, en diversas etapas del ciclo curricular. Los diferentes estilos de liderazgo directivo no dan razón a evidenciar un alza o baja sostenida de los aprendizajes en su totalidad, no siendo esos resultados sistematizados en el tiempo, y en veces erráticos en un posible pronóstico, lo que conlleva a indicar que el liderazgo, si bien es cierto, da un sello a cada establecimiento por el estilo impuesto, va enfocado mayormente a mejorar temas directamente relacionados a generalidades gestoras más que afianzar metodologías dentro del aula que pudiesen ir en búsqueda de resultados académicos que pudiesen ser sostenidas en el tiempo por los alumnos(as). Dentro de los aprendizajes medidos por la evaluación nacional SIMCE existe la posibilidad de subir anualmente pero también la posibilidad de bajar dichos aprendizajes es tan válida como la anterior conjetura, no siendo necesariamente el estilo que impone el Director(a) al gestionar el indicativo general de un alza o baja de un año otro de dichos aprendizajes. Los estilos de liderazgo no afectan puntualmente los resultados de aprendizaje, si bien es cierto el Director(a) guía de alguna forma equipos de trabajo dentro de los establecimientos, esto no conlleva el éxito dentro de evaluaciones externas, no así se podría aplicar esta teoría en los resultados dentro de cada escuela, lo que aun así también podría verse cuestionado. Los liderazgos que permanecen en el tiempo no acreditan los buenos resultados académicos, como tampoco los de corta duración, evidenciando una especie de azar en dicha evolución de dichos resultados, validando elementos no estudiados como fortalezas de buenos resultados, ejemplo: una generación distinta de alumnos(as), un estilo de enseñanza impuesta por un profesor(a) determinado que hizo efecto en ese grupo de alumnos(as) y no en otros, un grupo diferente de apoderados(as), etc.

Proponemos afianzar estrategias en común, sobre todo escuelas de un mismo Grupo Socio Económico (GSE). Variar la forma de liderar una escuela, en base a los nuevos escenarios que se encuentran en los diferentes momentos de la gestión Directiva y determinar tareas dentro de los diversos estamentos de una unidad educativa, para de alguna forma redefinir estilos de liderazgo marcados más a una teoría sistémica, en vez de apuntar al logro de resultados académicos, que, en estricto rigor, deben ser una de las metas de un colegio, derivando esta responsabilidad a equipos rigurosamente bosquejados para esto, por ejemplo, una UTP reformulada y especializada hacia lo que queremos.

\section{REFERENCIAS BIBLIOGRÁFICAS}

Ausbel, David. (2014). Teoría del aprendizaje significativo.

Bolívar, Antonio. (2010). El liderazgo educativo y su papel en la mejora: una revisión actual de sus posibilidades y limitaciones.

Bush, Tony y otros. (2017). Liderazgo educativo en la escuela, nueve miradas. Ediciones Universidad Diego Portales.

Castillo, Edelmira. (2003). El rigor metodológico en la investigación cualitativa.

Hernández Sampieri, Roberto. Metodología de la investigación. 
Jerico, Pilar. (2014). Los seis estilos de liderazgo de Daniel Goleman. Piqueras Gómez de Albacete, César. (2014). Seis estilos de liderazgo.

Ponce Vidal, Ricardo. (2008). El Liderazgo y su relación con el rendimiento académico. Universidad del Bio Bio.

Riffo Muñoz, Humberto. (2014). Gestión educacional y resultados académicos en escuelas municipales. Universidad Autónoma de Barcelona.

Sevilla Buitron, María. (2010). Principals and Student Achievement: Evidence from the Chilean Collective Performance Incentive Program Analysis of the 2005 through 2008 Cohorts. Ministerio de Educación de Chile.

\section{Web consultada:}

http://www.ideice.gob.do/index.php/programas/procesos-y-logros-de-aprendizaje http://www.nwlink.com/ donclark/leader/matrix.html

http://www.cesarpiqueras.com/6-estilos-de-liderazgo.-goleman/

http://es.slideshare.net/hteran1/enfoque-mixto http://metodologia02.blogspot.cl/p/operacionalizacionde-variables.html

http://metodologia02.blogspot.cl/p/metodos-de-la-inventigacion.html

http://es.slideshare.net/SandyDeLaOssa/mtodos-de-investigacion

https://es.scribd.com/doc/62764332/Procesamiento-y-analisis-de-informacion

http://www.monografias.com/trabajos71/definicion-estilos-liderazgo/definicion-estilosliderazgo.shtml\#ixzz 4bplyuoCe

http://www.monografias.com/trabajos71/definicion-estilos-liderazgo/definicion-estilos-liderazgo2.shtml\# estilosdea\#ixzz4bpMmaGaX

https://es.slideshare.net/JoanFernandoChipia/tipos-de-escalas-y-variables-estadsticas http://www.simce.cl/

http://www.simce.cl/ficha2016/?lista=1\&rbd=317\&establecimiento=escuela+las+rocas\&region=0\&comu na $=0$ \&nivel $=0$

http://www.simce.cl/ficha2016/?lista=1\&rbd=285\&establecimiento=liceo+mart+narea+diaz\&region=0\&c omuna $=0 \&$ nivel $=0$

http://www.simce.cl/ficha2016/?lista=1\&rbd=288\&establecimiento=escuela + jaon\&region=0\&comuna $=0$ \&nivel $=0$

http://www.simce.cl/ficha2016/?lista=1\&rbd=289\&establecimiento=escuela+esana\&region=0\&comuna= 0\&nivel $=0$

http://www.simce.cl/ficha2016/?lista=1\&rbd=293\&establecimiento=escuela+jose+papic+radnic\&region= $0 \&$ comuna $=0 \&$ nivel $=0$

http://www.simce.cl/ficha2016/?lista=1\&rbd=292\&establecimiento=escuela+republica+de+italia\&region $=0 \&$ comuna $=0$ \& nivel $=0$

http://www.simce.cl/ficha2016/?lista=1\&rbd=318\&establecimiento=escuela+edda+cuneo+donnagio\&reg ion $=0$ \& comuna $=0$ \& nivel $=0$

http://www.simce.cl/ficha2016/?lista=1\&rbd=304\&establecimiento=liceo+andres+sabella\&region=0\&co muna $=0 \&$ nivel $=0$

http://www.simce.cl/ficha2016/?lista=1\&rbd=12944\&establecimiento=reverendo+padre+patricio+cariola \&region $=0$ \& comuna $=0$ \&nivel $=0$

http://www.simce.cl/ficha2016/?lista=1\&rbd=291\&establecimiento=escuela+padre+gustavo+le+paige+w alque\&region $=0 \&$ comuna $=0 \&$ nivel $=0$

http://www.simce.cl/ficha2016/?lista=1\&rbd=298\&establecimiento=escuela+dario+salas+diaz\&region=0 \&comuna $=0 \&$ nivel $=0$

http://www.simce.cl/ficha2016/?lista=1\&rbd=313\&establecimiento=escuela+arturo+prat\&region=0\&co muna $=0 \&$ nivel $=0$

http://www.simce.cl/ficha2016/?lista=1\&rbd=320\&establecimiento=escuela+armando+carrera+gonzalez 
\&region $=0$ \& comuna $=0$ \& nivel $=0$

http://www.simce.cl/ficha2016/?lista=1\&rbd=12840\&establecimiento=escuela+juan+pablo+ii\&region=0

\&comuna $=0$ \&nivel $=0$

http://www.simce.cl/ficha2016/?lista=1\&rbd=326\&establecimiento=escuela+santiago+amengual+b.\&regi on $=0 \&$ comuna $=0 \&$ nivel $=0$

https://scielo.conicyt.cl/scielo.php?script=sci_arttext\&pid=S0718-45652015000200004

http://www.agenciaeducacion.cl/evaluaciones/que-es-el-simce/

http://www.educarchile.cl/ech/pro/app/detalle?id=194943

http://www.educarchile.cl/ech/pro/app/detalle?ID=107118

https://psicologiaymente.net/desarrollo/tipos-de-aprendizaje

http://www.psicoperspectivas.cl/index.php/psicoperspectivas/article/view/116/143 